\title{
CONTENTS
}

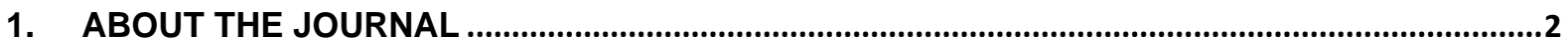

2. AIM AND SCOPE OF THE JOURNAL.............................................................................................

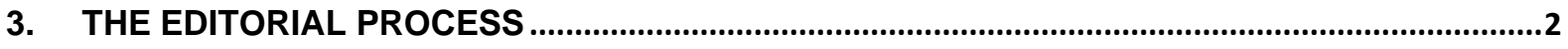

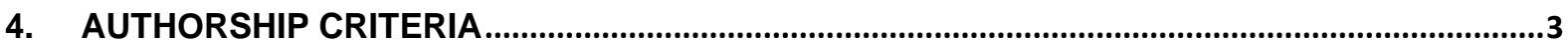

5. GENERAL GUIDELINES ...................................................................................................

6. ARTICLE TYPES ACCEPTED FOR PUBLICATION ...................................................................

7. MANUSCRIPT PREPARATION IN GENERAL...................................................................................4

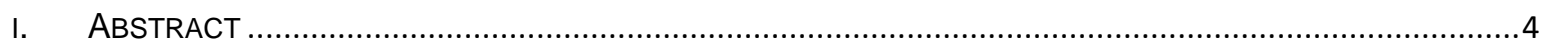

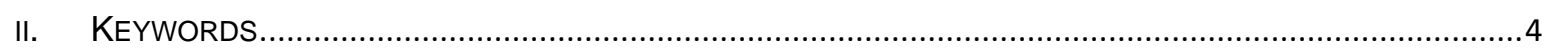

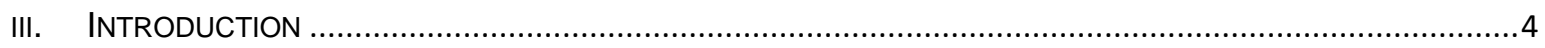

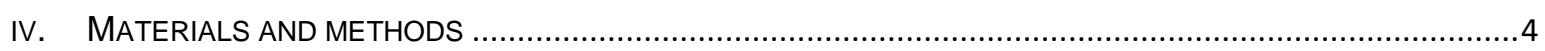

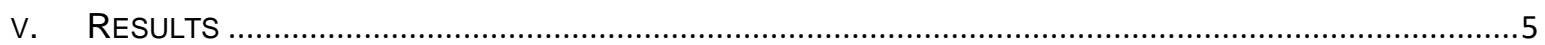

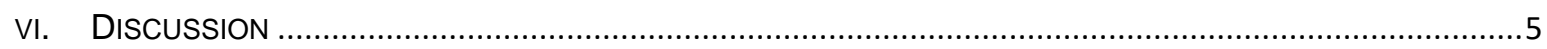

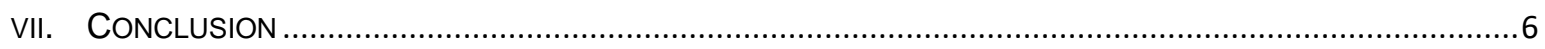

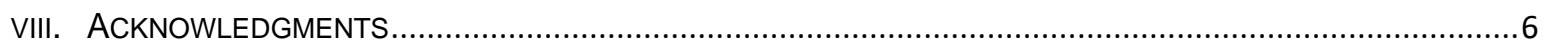

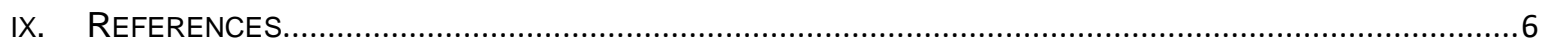

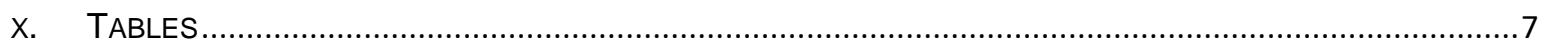

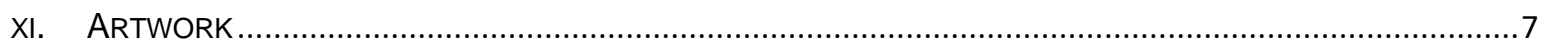

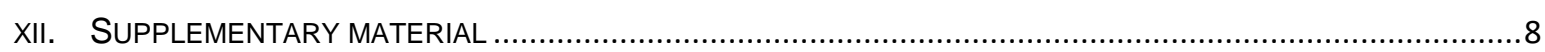

8. ARTICLE TYPE MANUSCRIPT PREPARATION....................................................................

9. SUBMISSION GUIDELINES ....................................................................................................10 


\section{About the Journal}

Geo Eye (GE) is a peer-reviewed, open-access, Bi-annual that aims to publish original articles, novel research and review articles encompassing subjects that fall under the wide spectrum of current trends of research on geography, geographical informatics and analysis around the world.

GE welcomes the articles to be considered for publication from a broad spectrum of Geography and geographical analysis disciplines, the coverage includes Geology, Geography, Geographical analysis, and Geo-informatics. GE accepts most types of manuscripts for consideration including original research papers, short communications, reviews, letters to the Editor and Editorials.

The journal is dedicated to the dissemination of knowledge related to the advancement in Basic and Scientific Research. The journal comprises a prestigious interdisciplinary editorial board that reflects the diversity of subjects covered in this journal.

GE is published under the emblem of Bangalore University. The online processing and hosting services rendered by Scientific Research Solution Pvt Ltd. The journal brings out Bi-annual issues and supports the open access policy for making scientific research accessible to one and all.

\section{Aim and Scope of the Journal}

Geo Eye (GE) aims to publish original and novelty research articles in the field of current trends of research on geography, geographical analysis and Geo-informatics. Our focus is to showcase the advancement in Science that can resolve the scientific problems and help to identify a new direction for further research. GE publishes original research articles, review articles, short communications, editorials, letters, and mini-reviews.

GE is dedicated to facilitating the scholarly researcher to publish their authentic scientific content and display it on various platforms with the help of digital technology. One of the major objectives of GE is to showcase scientific content in standard abstracting and indexing networks to make the content easily readable and findable to the scientific and non-scientific community.

GE editorial board is passionate to deliver the publication of content at a rapid turnaround time possible for reviewing and publishing and to disseminate the articles for research, teaching and reference purposes.

\section{The Editorial Process}

A manuscript will be reviewed for possible publication with the understanding that it is being submitted to GE alone at that point in time and has not been published anywhere, simultaneously submitted, or already accepted for publication elsewhere. All manuscripts received are duly acknowledged. 
On submission, editors review all submitted manuscripts. Manuscripts with insufficient originality, serious scientific or technical flaws, or lack of a significant message are rejected before proceeding for formal peer-review.

Manuscripts that are found suitable for publication in GE are sent to two or more expert reviewers. The journal follows a double-blind review process, where in the reviewers and authors are unaware of each other's identity.

Every manuscript is also assigned to a member of the editorial team, who based on the comments from the reviewers takes a final decision on the manuscript. The comments and suggestions (acceptance/rejection/revision in manuscript) received from reviewers are conveyed to the corresponding author. If required, the author is requested to provide a point by point response to reviewers' comments and submit a revised version of the manuscript.

Manuscripts accepted for publication are copy edited for grammar, punctuation, print style, and format. Page proofs are sent to the corresponding author.

The corresponding author is expected to return the corrected proofs within two days. The whole process of submission of the manuscript to final decision; sending and receiving proofs is completed online, with an average of 6 weeks duration.

As the journal is an under open access policy, and is freely available for readers without charges.

Correspondence with the authors by any members of editorial team except Editor-inChief and Editor is strictly prohibited.

\section{Authorship Criteria}

Authorship credit should be based only on substantial contributions to each of the three components mentioned below:

i. Concept and design of study or acquisition of data or analysis and interpretation of data;

ii. Drafting the article or revising it critically for important intellectual content; and

iii. Final approval of the version to be published.

Every manuscript should be accompanied by a contributor's form which describes about the contribution of each author towards the manuscript, with respect to the above authorship criteria. The originality of the manuscript has to be declared by the authors.

\section{General Guidelines}

i. Use only New Times Roman font.

ii. Prepare the manuscript in Microsoft Office Word with A4 size paper setting. 
iii. $\quad 18$ font for the title of the article.

iv. 14 font for the headings which should be bold.

v. $\quad 12$ font for sub-headings which should be bold.

vi. 12 font for the text.

vii. Do not use capital letters for title and sub-headings.

viii. Double-spacing should be maintained throughout the manuscript.

ix. Should number all of the pages of the manuscript consecutively, beginning with the title page.

\section{Article Types accepted for publication}

A manuscript with a research study relevant to the scope of the journal is expected from the authors. The study should be substantiated with appropriate study methodology, results, discussion and conclusion. Following are the types of manuscripts accepted for publication in the journal: Research article, Review article, Mini-review, Editorial, Short communication, Commentaries, Short notes. All the submitted manuscripts should follow the standard guidelines based on the types of manuscript, more details can be found in Table 1.

\section{Manuscript Preparation in General}

\section{i. Abstract}

The abstract should have the following subheadings: Objectives, Methods, Findings, Novelty and Keywords. The scientific terms appearing here for the first time should not be abbreviated. The word limit for the abstract is up to 250 words.

Please refer to the abstract word count in Table 2.

\section{ii. Keywords}

At the least 3 keywords and a maximum of 6 keywords can be included. They must be separated by commas to distinguish them. The first letter of all the keywords should be in capital letters

\section{iii. Introduction}

The introduction section should provide a context for your manuscript in brief. The introduction can include comprehensive information about the background, prior research in the field of study and objectives of research in the present study.

\section{iv. Materials and methods}


The materials and methods as the name suggests should detail the different materials and methods used in the study with relevant citations of previous studies. In this section, the scientific terms should be in the expanded form at their first appearance, they can be abbreviated in their subsequent appearances in the text.

If the manuscript includes trials on patients, the use of data of patients, confirmation about the consent from participant patients should be included. Information regarding the approval from the Institutional ethics committee should be part of this section as well. The reference to the methods should be cited with proper references for the readers to analyse and verify the reported results of the study.

This section should include all the experimental protocols gathered for the study for better understanding. The major methodology can be presented in the main manuscript, additional information about the methodology can be included as supplementary data.

The author is expected to maintain high stands of scientific integrity during the scripting of the manuscript. Recommendations as laid out by Committee on Publication Ethics (COPE) should be implemented in the manuscripts to improve the quality of the manuscript.

\section{v. Results}

The outcome of the experiments and study should be depicted in an easy to follow form for better understanding. The sequence of the results should be as per the experimental methods performed. The results should be aligned to the tables, graphs or figures for analysis and verification by the reader. The repetition of data in the tables and figures should be avoided.

Additional information related to the results can be provided as supplementary materials.

\section{vi. Discussion}

The discussion should include the analysis of the methods and the results obtained from them. The inference or the significance of the results should be described in detail. This can include content to prove that the improvisation from the previous work cited and its significant outcomes to prove the achievement of the objectives. Caution should be excised not to repeat the information given under introduction and results. 
vii. Conclusion

The conclusion should summarise an analytical outcome of the present studies and the results obtained. The significance of the results and discussion should be presented in brief to conclude the study. A scope for further research should be included as a suggestion to continue or steer future research in the present field of study.

\section{viii. Acknowledgments}

This section provides you with an opportunity to acknowledge individuals for their contribution and support, extended for the study but are not listed as one of the authors. The support extended in the form of funds or research studies from different institutes or agencies can be attributed here.

\section{ix. References}

The references are listed as per the alphabetical order of the first authors in the references. They are numbered using Arabic numerals as 1). The citations in the text should be in square brackets as depicted [1]. The cited numbered references should be depicted after the full stop or period of the sentence. All the cited references should be listed in the reference section of the manuscript.

The reference of the manuscripts listed should be formatted as per the following examples.

\section{For Journal}

Authors Surname, Initial; Article Title. Journal name, Year of Publication; Volume (Issue): Page No.

Example: Ochel HJ, Gademann G, Rocken C, Wordehoff H; Effects of imatinib mesylate on adenoid cystic carcinomas. Anticancer Research, 2005; 25(5): 36593664.

Note that all the authors listed until 6 authors of a single reference can be listed as per the aforementioned style.

After the first six authors, the authors to be listed should be depicted by et al. The references in the manuscript can be accepted as long as all the references are consistent with one of the reference styles accepted worldwide. 


\section{For a book and other Monograph}

Authors/Editors Surname Initial; Book Name. Edition, Publisher, Place of publication, Year of publication: Page No.

Example: Kar A; An Introduction to Medicinal Chemistry. 4th edition, New Age International Publishers, New Delhi, 2007: 199-202.

\section{Book Chapter}

Authors Surname Initial; Chapter Name. In Book Name, Edition, Editors Surname Initial, Publisher, Place of publication, Year of publication: Page No.

Example: Mandell GL, Petri WA; Antimicrobial agents: Penicillins, Cephalosporins and other beta lactam antibiotics. In Goodman and Gillman's: The Pharmacological basis of Therapeutics. 9th Edition, Hardman JG, Limbard LE editors, McGraw-Hill, New York, 1996:1073-1101.

\section{For Thesis}

Authors Surname Initial; Thesis Title. University, Place, Year: Page No.

Example: Sharma SK; A Survey on Anticancer drugs in Delhi. Indian University, New Delhi, 2012: 40-50.

\section{Website link}

Title of the Topic, available from Link and accessed month and year.

Example: Herbal Medicine. Available from

http://www.nlm.nih.gov/medlineplus/herbalmedicine.html (accessed on Jan 2016)

\section{x. Tables}

The tables should be submitted as editable text and not as images. Tables should be placed as a separate document. Number the tables consecutively as per their appearance in the text and place any reference notes below the table body. The table can be named as 'Table 1. "Table name", the table numbers should be in Arabic numerals and should be cited near the appropriate text.

\section{xi. Artwork}

The artwork should be of uniform sizing and lettering for the manuscript. The preferred font styles are Times New Roman, font size 12 and double (2.0) spaced between the lines. Number the illustrations/figures/pictures according to their sequence in the text. 
Please include all the figures or images with figures legends in a single and separate file numbered in Arabic numerals and should be cited at the appropriate text. The TIFF (or JPG), colour or grayscale photographs (halftones) should be a minimum of 300 dpi. Disproportionately large graphics should not be submitted. Avoid providing files that are optimized for screen use (e.g., GIF, BMP, PICT, WPG) or low-resolution images and graphics that are disproportionately large for the content. Please make sure that artwork files are in an acceptable format [TIFF (or JPEG), EPS (or PDF), or MS Office files] and with the correct resolution.

\section{xii. Supplementary material}

The data in the form of tables, figures and graphs that can be considered for verification by the readers or is further reading for the content in the manuscript are considered supplementary. The supplementary material is submitted as separate documents during the submission that will be available online only.

The Submitted supplementary materials are published exactly as they are received (Excel or PowerPoint files will appear as such online). Referencing of the supplementary material in the manuscript should be similar to other documents, except that the title should be sufficed by the word supplementary e.g. supplementary table, supplementary figure, etc. along with numbers in Arabic numerals.

Table 1: Guidelines for manuscript preparation

Guidelines based on types of study

Strengthening the Reporting of Observational

Studies in Epidemiology (STROBE)

\section{Source web link}

https://www.strobe-

statement.org/index.php?id=strobe-home http://www.prisma-statement.org/

staternent
Preferred Reporting Items for Systematic Reviews and Meta-Analyses (PRISMA) 
Table 2: Style guide for manuscripts

\begin{tabular}{cccccc}
\hline Article Type & $\begin{array}{c}\text { Abstract - } \\
\text { word count }\end{array}$ & $\begin{array}{c}\text { Body of the } \\
\text { manuscript - References in } \\
\text { word count }\end{array}$ & Tables & Figures \\
\hline Research article & $150-250$ & Max 3000 & Max 40 & 3 & 3 \\
\hline Review article & Max 300 & Max 8000 & Max 100 & 4 & 6 \\
\hline Mini-review & Max 200 & Max 3000 & Max 30 & 2 & 2 \\
\hline Editorial & NA & Max 1000 & NA & 1 & 1 \\
\hline $\begin{array}{c}\text { Short } \\
\text { Communication }\end{array}$ & Max 150 & Max 1500 & Max 12 & 2 & 1 \\
\hline Commentaries & NA & Max 400 & Max 4 & NA & NA \\
\hline Short notes & NA & Max 300 & Max 2 & NA & NA \\
\hline
\end{tabular}

Note: If the number of tables or figures is less than mentioned in the tables, either tables or figures can be added to compensate for the maximum as mentioned in the table.

\section{Article Type Manuscript Preparation}

- $\quad$ Language and Page Format

Manuscript need to be in English language only and should be prepared in Microsoft Word with a font size of 12 and in Times New Roman font style on A4 size document leaving the margins of 1 inch on all four sides in single column. The line spacing should be double spaced including references and tables.

- $\quad$ Title page and Authors Details for all type of articles

Title page should contain the following information

Title: Precise title of the article

Author Name(s): Full name of each authors

Affiliation(s): Authors affiliation with address

Corresponding Author: Name, Affiliation, functional Contact Number and Email ID

- $\quad$ Research Articles

Manuscript should be under the following headings

Abstract: Should be in unstructured format with the word limit of not more than 250 words. 
Keywords: It should contain up to 4-6 terms related to the work.

Introduction: It should represent the background, literatures survey, scope and significance of the present work.

Materials and Methods: It should contain information regarding the materials and methods used to carry out the present research work.

Results and Discussions: This section should contain the obtained results, interpretation of results and discussion on the findings.

Conclusion: In this section, outcome of the research need to be explained.

Acknowledgment: It should contain the brief information regarding research funding, grants or the assistance of colleagues or the institutions.

References: All the references should be arrange at the end of manuscript in order of their citation in the text and references should be cited in superscript format in the text.

- $\quad$ Review Article

For a review article, authors should have done extensive work on the subject reviewed or considered experts in the respective domain. The manuscript should have an Abstract of 250 words possessing an accurate summary of the work reviewed. The section headings and sub headings would depend upon the type of subject considered.

Acknowledgment: It should contain the brief information regarding research funding, grants or the assistance of colleagues or the institutions.

References: All the references should be arrange at the end of manuscript in order of their citation in the text and references should be cited in superscript format in the text.

\section{Article Submission Guidelines}

All the manuscripts must be submitted on-line through the journal website 'submit manuscript' link. The Author has to register by filling up all the information in a web form. A new user ID and password will be assigned which can be used to login and submit manuscripts. The author can follow up the manuscripts for publication through the same system. 
After the manuscript is readied according to the guidelines provided to the author. The manuscript can be prepared for submission by providing the necessary documents as listed below:

1. Cover letter: This is the letter addressed by the author to the Editor-in-Chief of the journal. The body of the letter may contain the title, type of manuscript, the importance of the study reported in the manuscript and the reason for choosing the journal for publication.

2. Title page: This document should contain the title, running title, authors, their affiliations, email IDs, corresponding author, correspondence address, phone number, the word count of the abstract, word count of the article excluding the abstract and references, number of figures, number of tables and supplementary material.

3. Article file: This should contain the article with abstract, materials and methods, results, discussion, conclusion and references.

4. Tables: All the tables should be with titles and footnotes (if necessary), this document should be provided in editable MS-Word format.

5. Figures: All the figures and images should be numbered (e.g. Figure 1) followed by figure legends.

6. Supplementary material: This document may contain additional parts of a manuscript. They may be in the form of tables, figures and datasets, these may not appear as part of the manuscript and it will be available in the online format.

7. Copyright transfer form: A document for authorization of copyrights transfer.

All these documents may not be required for all types of manuscripts during submission. Read the instructions thoroughly before commencing the submission of the manuscript. We look forward to your association with all your publications in the future.

For any query regarding manuscript/article/document submission, please feel free to contact our journal editorial team by filling the contact form with details of your query.

\section{Contact}

Dr. Ashok D Hanjagi

Editor in Chief

Professor, Department of Geography

Bangalore University, Bangalore

Email: ashokhanjagi@bub.ernet.in 